\title{
FUZZY-SITUATIONAL DIAGNOSTICS OF TECHNOLOGICAL SAFETY OF PETROCHEMICAL PLANTS
}

\author{
Sidikov Isamidin Hakimovich \\ Tashkent State Technical University \\ Department of Information Technology in \\ Control, University st.2, 100095 Tashkent, \\ Republic of Uzbekistan
}

\author{
Atajonov Muhiddin Odiljonovich \\ Tashkent State Technical University \\ Department of Information Technology in Control, \\ University st.2, 100095 Tashkent, \\ Republic of Uzbekistan
}

\author{
Atajonova Saida Baratalievna \\ Andijan Machine Building Institute \\ Automation of Processes Production, \\ 170019. Andijan. Republic of Uzbekistan
}

\begin{abstract}
The article will discuss the issues of fuzzy modeling and it will provide the analysis of technology-related situations for ensuring the safety of complex industrial facilities in line with the decision-making process of their management. On the basis of theory of fuzzy sets and fuzzy logic, the process of making decisions under the conditions of uncertainty and uncertainty of the initial information will be studied.

In addition, the study will look at the problems in diagnostics and control of technical safety at chemical plants and chemical industry, in general. It has been proven that the diagnostic system of chemical processes lies at the core of the modern safety process and control system of chemical production. A great deal of research has been done on a number of general features within the creation and use of aforementioned diagnostic systems in chemical processes.
\end{abstract}

The research has employed the formal method in which the functioning dynamics of petrochemical facilities is analyzed based on the theory of fuzzy sets and fuzzy logic. This method allows to develop a set of measures aimed at managing the technical safety of petrochemical facilities and, consequently, the reduction of loss and the increase of efficiency among the personnel. This the efficiency of service personnel can be achieved by improving the state of performance and predicting technology-related failures within equipment and control systems.

Keywords - Information networks, diagnostics, process safety, soft computing, fuzzy set theory, fuzzy logic, fuzzy model, fuzzy decision making, complex industrial facilities

\section{INTRODUCTION}

The automatic classification method of the states of complex object is considered. This method based on the algorithm of clustering fuzzy c-average values and on the algorithm of situation logical deduction for control in conditions with fuzzy source information [1].

One of the main directions in the organization of industrial production is to ensure the safety of technical processes (TP), which is largely determined by the compliance with safety requirements, the timely determination of the conditions, and the diagnostics of technical equipment and units, as well as the effectiveness of management in various situations that occur in the technical cycle [2].

\section{RESEARCH METHODS}

The thorough analysis of the principles in constructing diagnostic systems and managing technical safety with petrochemical plants and facilities leads to the conclusion that the presence of a big number of input and output characterizing the state of the aforementioned system in an ambiguous manner is the significant feature, on which, however, there is no or a very limited knowledge about the physio - chemical process parameters [2,3].

In addition, there are difficulties in solving the problems related to diagnostics and management of technical safety in complicated technical systems that occur in various emergency situations. These difficulties arise due to the fact that the most of decisions are made facing the conditions of uncertainty and fuzziness of the initial information, and there are practically no possibilities of using the existing deterministic stochastic models [1-4].

An analysis of the principles of constructing diagnostic systems and managing technological safety 


\section{International Journal of Engineering Applied Sciences and Technology, 2019 Vol. 4, Issue 7, ISSN No. 2455-2143, Pages 182-186 \\ Published Online November 2019 in IJEAST (http://www.ijeast.com)}

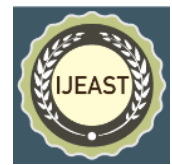

with petrochemical plants and complexes leads to the conclusion that a significant feature of this class of systems is the presence of a large number of input and output parameters that characterize the state of the system in an ambiguous manner, the absence or incompleteness of knowledge about the physicochemical process parameters [2-5]. Additional difficulties in solving the problems of diagnosing and managing technological safety with complex technical systems in various emergency situations arise due to the fact that decisions are mainly made in the conditions of uncertainty and fuzziness of the initial information and there are practically no possibilities to use the existing deterministic-stochastic models [3-8].

\section{CURRENT RESEARCH}

Based on the above, it can be said that for the effective management of complicated technical processes described above there is an urgent need for the development of new methods, models, and approaches. These scientific approaches can assist in the assessment of the status of technical equipment and related processes, the algorithms and methods and for ensuring the safe functioning of facilities in various industrial and technical situations, and these models need to be based on the use of modern information technologies as well as intelligent decision support tools [4-9].

The present research introduces one of the possible approaches to situational analysis and assessment of the condition of control objects as well as the methods of situational inference in the cases of uncertainty and fuzziness of the initial information.

The result of the analysis conducted for the current research should serve as an assessment of possible levels of danger. Therefore, the paper proposes to quantitatively assess the level of danger as the degree of remoteness of the current state of the process from the area of the security center, in which the functioning of the process could be regarded as the safest [8-10].

A systematized approach in finding solutions to the problems of creating systems in order to prevent the development of potentially dangerous situations has proven itself to be efficient in a number of enterprises. The interruptions caused by partial equipment failure can be significantly reduced. In this case, the programming software needs to be developed in accordance with the emergence of new tasks. This could be an intensive way of industry development, which differs from the extensive one, where the solution to the problem was sought by constantly attracting new resources; the extensive way does not help to reduce the costs of maintaining the regimes [3-12].

The partial equipment failure discussed above as well as the violations of technical conditions can be prevented by focusing on finding scientific solutions and on making attempts to generalize the computerization of the control system [12-15].

\section{FINDING SOLUTIONS}

Let us assume that the set of possible states of the control object is defined by a set $\mathrm{S}$ of referenced fuzzy situations. It is also assumed that the set of referenced situations $\mathrm{S}$ is complete. Based on the expert information, each fuzzy situation is associated with a control solution, where $\mathrm{R}$ is the set of control decisions used to control the object.

Here, the fuzzy situational logical conclusion is implemented to recognizing the input fuzzy situation describing, that describes the current state of the control object and issuing the corresponding control solution from the set $\mathrm{R}$. To recognize the fuzzy situation, two methods can be proposed: the "nearest neighbor" method in the space of referenced fuzzy situations and the second method of issuing control decisions where all referenced situations are taken into account.

As a measure of the similarity of fuzzy situations, the degree of fuzzy inclusion of fuzzy situations and the degree of fuzzy equality are most preferred. Both of these measures consist in calculating the degree of similarity in the interval $[0 ; 1]$. The greatest degree of similarity is 1 , the smallest is 0 . The degree of similarity of 0.5 means complete uncertainty.

Consider the technological process, given in general terms as follows: $T P=\left(M^{T O}, R^{M}, S\right)$,

where: $M^{T O}=\left\{M_{1}^{T O}, M_{2}^{T O}, \cdots, M_{\mathrm{n}}^{T O}\right\}$

many models of technological equipment and assemblies (technological system); $R^{M}$ - a lot of connections between objects; $S$ - is the set of states of objects.

The functioning of any TP can be considered as a sequence of state changes over a $S_{t} \in S=\left\{S_{1}, S_{2}, \cdots S_{n}\right\} \quad$ certain time interval $\left[\mathrm{t}_{0}, \mathrm{t}_{\mathrm{k}}\right]$. The state $S_{t}$ of TP at each moment of time is $\mathrm{t}^{*} \in\left[\mathrm{t}_{0}, \mathrm{t}_{\mathrm{k}}\right]$ characterized by a set of parameters: $Y_{t^{*}}=\left\langle\mathrm{Y}_{i}^{T P}, Y_{j}^{T O}, \mathrm{Y}_{1}^{\mathrm{SC}}\right\rangle$, where: $\mathrm{Y}_{i}^{T P}, \quad \mathrm{i}=\overline{1, \mathrm{I}}$ parameters of the state of technology of this process; $\mathrm{Y}_{j}^{T O}, \quad \mathrm{i}=\overline{1, \mathrm{~J}}$ - equipment status parameters; $\mathrm{Y}_{1}^{S C}, \quad 1=\overline{1, \mathrm{~L}}$ - control system status parameters.

The technical processes can be subject to restrictions on regular functioning, $\Psi\left\{\overline{\mathrm{Y}^{T P}}, \overline{Y^{T O}}, \overline{\mathrm{Y}^{\mathrm{SC}}}\right\} \leq 0$, depending on the set of parameters $\left\{\mathrm{Y}_{i}^{T P}, Y_{j}^{T O}, \mathrm{Y}_{1}^{\mathrm{SC}}\right\}$. Going beyond these restrictions means the transition of technical processes to an emergency situation. Thus, these restrictions divide the space of all states in which technical processes can be located into two sets: $\mathrm{S}^{\mathrm{OC}}$ - a lot of dangerous states and 


\section{International Journal of Engineering Applied Sciences and Technology, 2019 \\ Vol. 4, Issue 7, ISSN No. 2455-2143, Pages 182-186 \\ Published Online November 2019 in IJEAST (http://www.ijeast.com)}

$\mathrm{S}^{\mathrm{PC}}$ a lot of safe (working) states, i.e. $S=S^{O C} \cup S^{P C}, S^{O C} \cap S^{P C}=\varnothing$. In turn, the set of dangerous states can be divided into two disjoint subsets: $S^{O C}=S^{O C_{1}} \cup S^{O C_{2}}, S^{O C_{1}} \cap S^{O C_{2}}=\varnothing$, where $S^{O C_{1}}$ are the subsets of dangerous $S^{O C_{2}}$ states of technical processes in the zone of warning and maximum permissible values of technological parameters.

In a variety of safe conditions, the area or point at which the technical processes operation is the safest is the area of technological safety center of greatest interest $S_{0} \in S^{P C}$. If the technological process is characterized by hazardous parameters, all values of which lie in the range of permissible values $S_{0}$, the current hazard can be considered zero. If one or several parameters go into the zone of dangerous values $S^{O C_{1}}$, the current danger increases, and it will increase as the parameters approach the zone of critical values $S^{O C_{2}}$. It is intuitively clear that the current danger of the process must depend on the multitude of the number of hazardous parameters simultaneously in the zone $S^{O C_{1}}$, on the degree of approach of each parameter to the zone $S^{O C_{2}}$ and on the degree of influence of each hazardous parameter on the possibility of an emergency.

Suppose that each set of parameters $y_{i} \in Y, Y=\left(y_{1}, y_{2}, \cdots, y_{p}\right) \quad$ whose values describe the state of the object corresponds to linguistic variables $<y_{i}, T_{i}, D_{i}>$, where $T_{i}=\left\{T_{1}^{i}, T_{2}^{i}, \ldots, T_{m}^{i}\right\}$ is the term-set of linguistic variables (LV), $y_{i}$ is the set of linguistic values of the attribute, $m_{i}$ is the number of values of the attribute; $D_{i}$ - the base set of the attribute $y_{i}$. To describe $T_{j}^{i}\left(i \in L=\left\{1,2, \ldots, m_{i}\right\}\right)$ the terms corresponding to the values of the attribute $y_{i}$, fuzzy variables are used, $<T_{j}^{i}, D_{i}, \widetilde{C}_{j}^{i}>$ i.e. value $T_{j}$ - is described by a fuzzy set in the base set $D_{i}$ :

$$
\tilde{C}_{j}^{i}=\left\{<\mu_{C_{j}^{i}}(d) / d>\right\}, d \in D_{i}
$$

Then the fuzzy situations that arise during the functioning of the system can be represented as a fuzzy set of the second level:

In this case, the task of assessing technological safety and making rational decisions in various emergency situations, in essence, can be formulated as the task of determining and classifying fuzzy situations
$S_{i} \in S=\left\{S_{1}, S_{2}, \cdots S_{n}\right\}$ using the situational inference method. Then, comparing the input fuzzy situation $\tilde{S}_{0}$ with each fuzzy situation from a certain set of typical fuzzy situations $\tilde{S}=\left\{\tilde{S}_{1}, \tilde{S}_{2}, \ldots, \tilde{S}_{N}\right\}$, you can determine the optimal response alternative (sequence of actions), which transfers the system from state $S_{t}^{0}$ to $\mathrm{S}_{t^{\prime}}^{*}$, where a lot of system parameters $\left\{\mathrm{Y}_{i^{*}}^{T P}, Y_{j^{*}}^{T O}, \mathrm{Y}_{1^{*}}^{\mathrm{SC}}\right\} \quad$ characterize the "center" of technological safety indicators of the production process.

\section{THE CONCEPT OF SOLVING THE PROBLEM}

Let be the set of states of an object $\mu_{1}, \mu_{2}, \ldots, \mu_{k}$, membership functions for fuzzy clusters $F_{1}, F_{2}, \ldots, F_{k}$. Fuzzy clusters form a fuzzy coverage of a set if and only if:

$$
\mu_{1}(y)+\mu_{2}(y)+\ldots+\mu_{k}(y) \geq 1, \forall_{y} \in Y
$$

The quality of the fuzzy coating can be estimated using the following characteristics:

$$
J_{(\mu)}=\min \sum_{i=1}^{k} \sum_{y \in Y}\left(\mu_{i}(y)\right)^{2}\left\|y-V_{i}\right\|^{2},
$$

where $V_{1}, V_{2}, \ldots, V_{k}$ - are the centers of the clusters, $V_{i} \in L$ is the vector space with the norm \|\| generated by the scalar product, $J_{(\mu)}$ - determines the standard deviations of states $Y$ from relative to the centers.

Clustering algorithm for fuzzy situations.

Step 1. Based on the clustering algorithm, an initial classification of states by clusters is performed, $\mu_{i}(y), i=\overline{1, k}$ and characterizes the proximity of the state $y$ to the center of the $\mathrm{i}$-th cluster.

Step 2. Cluster centers are specified using the formula:

$$
V_{i}=\sum_{y \in Y}\left(\mu_{i}(y)\right)^{2} y / \sum_{y \in Y}\left(\mu_{i}(y)\right)^{2}, i=\overline{1, k, y \in Y \subset L}
$$

Step 3. A new coating $F_{1}, F_{2}, \ldots, F_{k}$ is being constructed $\left(\mu_{1}, \mu_{2}, \ldots, \mu_{k}\right)$, described using the rule:

$$
\mu_{i}(y)=\frac{1}{\left\|y-V_{i}\right\|^{2}} / \sum_{j=1}^{k} \frac{1}{\left\|y-V_{1}\right\|^{2}}
$$

Step 4. The deviations of $\mathrm{T}$ value from $\mu=\left(\mu_{1}, \mu_{2}, \ldots, \mu_{k}\right)$ are $\mu$ calculated. If $\delta \leq \varepsilon \quad$ - a 


\section{International Journal of Engineering Applied Sciences and Technology, 2019 \\ Vol. 4, Issue 7, ISSN No. 2455-2143, Pages 182-186 \\ Published Online November 2019 in IJEAST (http://www.ijeast.com)}

certain threshold, then the algorithm is completed; otherwise, go to step 2.

The size of the training sample needed to build the coverage of the state space of an object by clusters refers to the number of uncertain parameters that must be determined during the training.

Using the fuzzy clustering algorithm, it is determined that the state does not belong to cluster $F$, but to what extent it belongs to $F$.

Suppose that in the process of cluster analysis, a fuzzy coverage of the state space of the object with fuzzy clusters $F_{1}, F_{2}, \ldots, F_{k}$ was built, and the current state of the object $S_{0}$ is fed to the input of the information system (IS). In the process of $S_{0}$ recognition, a set of values is determined that $\mu_{01}, \mu_{02}, \ldots, \mu_{0 k}$ characterizes the correspondence of the $S_{0}$ state to each cluster $F_{i}, i=\overline{1, k}$. If the obtained values of $\mu_{01}$ lead to the fulfillment of a relation of the form $\mu_{01}, \mu_{02}+\ldots+\mu_{0 k} \leq 1$ or maxi $\mu_{01} \leq T$, where $\mathrm{i}$ - is the cluster number, $\mathrm{T}$ - is a certain threshold, then a decision is made to create a new fuzzy cluster $F_{k+1}$, whose center is $S_{0}$.

\section{SITUATIONAL INFERENCE}

The tasks of situational choice in the general case can be considered as the tasks of determining the current state of the control object $\tilde{S}^{*} t^{*}$ and comparing it with each fuzzy situation from a certain set of typical fuzzy situations $S=\left\{\tilde{S}_{1}, \tilde{S}_{2}, \ldots, \tilde{S}_{N}\right\}$.

For this, as a measure of proximity between the current fuzzy situation $\tilde{S}^{*} t^{*}$ and the $\tilde{S}_{0}$ situation corresponding to the center of technological safety, we will use the following two criteria: the degree of fuzzy inclusion and the degree of fuzzy equality.

$$
\text { Let } \tilde{S}_{i}=\left\{<\mu_{S i}(y) / y>\right\}, \tilde{S}_{j}=\left\{<\mu_{S i}(y) / y>\right\}
$$

$(y \in Y)$ there be some situations. Then the degree of inclusion of the situation $\tilde{S}_{i}$ in the situation $\tilde{S}_{j}$ is determined by the expression:

$$
v\left(\tilde{S}_{i}, \widetilde{S}_{j}\right)=\&_{y \in Y} v\left(\mu_{S_{j}}(y), \mu_{S_{j}}(y)\right)
$$

where

$$
\begin{aligned}
v\left(\mu_{S_{i}}(y), \mu_{S_{j}}(y)\right) & =\underset{l \in L}{\&}\left(\mu_{\mu S i}\left(T_{i}^{l}\right) \rightarrow \mu_{\mu S j}\left(T_{j}^{l}\right)\right) \\
\mu_{\mu S i}\left(T_{i}^{l}\right) \rightarrow \mu_{\mu S j}\left(T_{j}^{l}\right) & =\max \left\{1-\mu_{\mu S i}\left(T_{i}^{l}\right), \mu_{\mu S j}\left(T_{j}^{l}\right)\right\}
\end{aligned}
$$

Here $v\left(\mu_{S i}(y), \mu_{S j}(y)\right)$ is the degree to which the fuzzy set $\mu_{S i}(y)$ is included in the fuzzy set $\mu_{S i}(y)$.

It is believed that the situation $\tilde{S}_{i}$ is not clearly included in the situation $\tilde{S}_{j}, \widetilde{S}_{j}, \widetilde{S}_{i} \subseteq \tilde{S}_{j}$, if the degree of inclusion $\tilde{S}_{i}$ in $\tilde{S}_{j}$ is not less than some inclusion threshold determined $\alpha^{*}{ }_{\text {inc }}$ by the control conditions, i.e. $v\left(\tilde{S}_{i}, \tilde{S}_{j}\right) \geq \alpha^{*}{ }_{i n c}$. The determination of the inclusion threshold point mainly depends on the properties of the control object and the requirements for the quality of control.

The existence of two mutual inclusion of situations $\tilde{S}_{i}$ also $\tilde{S}_{j}$ means that at the threshold of inclusion, the $\alpha^{*}{ }_{\text {inc }}$ situations $\tilde{S}_{i}$ and $\tilde{S}_{j}$ are approximately the same. This similarity of situations is called fuzzy equality, and the degree of fuzzy equality of $\mu\left(\tilde{S}_{i}, \tilde{S}_{j}\right)$ situations $\tilde{S}_{i}$ is $\tilde{S}_{j}$ determined as follows:

$$
\mu\left(\tilde{S}_{i}, \tilde{S}_{j}\right)=\underset{y \in Y}{\&} \mu\left(\mu_{S i}(y),\left(\mu_{S j}(y)\right)\right.
$$

Where

$$
\begin{array}{r}
\mu\left(\mu_{S i}(y),\left(\mu_{S j}(y)\right)=\underset{l \in L}{\&}\left(\left(\mu_{\mu S i}\left(T_{i}^{l}\right) \rightarrow\right.\right.\right. \\
\left.\mu_{\mu S j}\left(T_{j}^{l}\right)\right) \&\left(\mu_{\mu S j}\left(T_{i}^{l}\right) \rightarrow \mu_{\mu S i}\left(T_{j}^{l}\right)\right)
\end{array}
$$

It is believed that the situations $\tilde{S}_{i}$ and $\tilde{S}_{j}$ are fuzzy equal, $\tilde{S}_{i} \approx \tilde{S}_{j}$,

if, $\mu\left(\tilde{S}_{i}, \tilde{S}_{j}\right) \geq \alpha, \alpha \in[0.6 ; 1]$ where $\alpha-$ is a certain threshold of fuzzy equality of situations.

If situations $\tilde{S}_{i}$ are $\tilde{S}_{j}$ described by p signs, then for their $(p-q)$ - generality, a fuzzy equality of $p-q$ signs from the set is sufficient.

If the signs with which the control object is described are independent of each other, then from some situation $\tilde{S}_{i}$ we can go into any situation $\tilde{S}_{j}$ that has $(p-q)$ - commonality with the situation $\tilde{S}_{i}$, using no more than q local (acting on the value of only one sign ) controls. Then the degree of $(p-q)$ is the generality of $k_{p-q}\left(\tilde{S}_{i}, \tilde{S}_{j}\right)$ situations $\tilde{S}_{i}$ and $\tilde{S}_{j}$ is determined by the expression

$$
k_{p-q}\left(\tilde{S}_{i}, \tilde{S}_{j}\right)=\underset{y \in Y / Y_{q}}{\&} \mu\left(\mu_{S i}(y), \mu_{S j}(y)\right),
$$




\section{International Journal of Engineering Applied Sciences and Technology, 2019 Vol. 4, Issue 7, ISSN No. 2455-2143, Pages 182-186 \\ Published Online November 2019 in IJEAST (http://www.ijeast.com)}

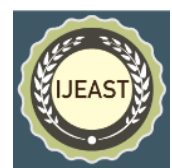

where, $\left|Y_{q}\right| \leq q$ the attribute $y_{k}$ belongs to $Y_{q}$ if $\mu\left(\mu_{S i}\left(y_{k}\right), \mu_{S j}\left(y_{k}\right)<\alpha, \quad\right.$ for $\quad Y_{q}=\phi$, the

situations $\tilde{S}_{i}$ and $\tilde{S}_{j}$ are fuzzy equal.

\section{RESULT}

The main problem of industrial diagnostic systems is the timely detection of violations that lead to emergency situations. In this regard, the urgent task of finding the primary fault and the task of analyzing the possible negative impact of this fault on the next in the chain of nodes and devices.

In this paper, it is proposed to use fuzzy binary relations as a mathematical apparatus for constructing a qualitative picture of the development of hazards, which make it possible to approach the question of creating chains of interference of hazards from more formal positions and to expand the multitude of searches for possible options for the development of hazards. In addition, this method should use expert judgment.

\section{CONCLUSION}

The paper considers mathematical models used in the problems of diagnosing the states of technological systems: structural-logical and causal models, statespace models, and interval parametric models. The problems of diagnosing conditions and controlling the technological safety of chemical plants are considered. It is shown that the core of the modern process safety control system for chemical production is the diagnostic system for the state of the chemical process, a number of general features of the creation and use of diagnostic systems for the chemical process are given.

Thus, the use of the proposed methodology for formalizing the dynamics of the functioning of petrochemical complexes based on the theory of fuzzy sets and fuzzy logic allows us to develop a set of measures aimed at managing the technological safety of petrochemical facilities and, accordingly, to reduce losses and increase the efficiency of staff by improving the condition operability and failure forecasting of technology, equipment and control systems.

\section{REFERENCES}

[1] V. S. Simankov, A.V. Shopin, "Situational Control of a Complex Object in the Conditions of Fuzzy Initial Information", Krasnodar works of FORA. № 9. Pp.116-120, 2004.

[2] D.P. Vent, A.E. Prophets, N.A. Toichkin, "Mathematical models used in diagnostics of technological systems". № 3, Pp. 1-20. 2015.

[3] I.H. Sidikov, D. Umurzakova, Adaptive Neurofuzzy Regulating System of the temperature
Mode of the Drum Boiler, IJARSET, Volume 6, Issue 1, January, Pp. 7869-7873, 2019.

A.F. Egorov, T.V. Savitckaya, "Methods and models of risk analysis and safety chemical technology control", Basis of theory chemical technology. Tom 44, №3. Pp.341-353, 2010.

[5] I.H. Sidikov, D. Umurzakova, Mathematical Modeling of Transient Processes of the Automatic Control System of Water Level in the Steam Generator. UJME., 7(4): DOI: 10.13189/ujme.2019.070401.pp. 139-146, 2019.

[6] N.G. Yarushkina, "Basis of theory fuzzy and hybrid systems". Moscow: Publishing house «Financial and statistic». Pp.- 320, 2004.

[7] R.A. Aliyev, R.R. Aliyev, "Theory of intellectual systems". Baku: Publishing house «Chashiogly», Pp. -720, 2001.

[8] A.V. Andreychikov, O.N. Andreychikova, "Intellectual information systems", Moscow: Publishing house «Financial and statistic». Pp.2004. - 424.

[9] A.A. Uskov, "Intellectual technology of control. Artificial neural network and fuzzy logic". Moscow: Publishing house «Hot line - telekom». Pp. 2004. -143.

[10] A. Riid, Transparent Fuzzy systems: Modeling and Control. Pp. 2002. -227.

[11] Deng Yong, Shi Wenkang, A modified aggregation of fuzzy opinions under group decision making. // J.of Computers and Systems Sciences International. V. 42. 2003.

[12] Z.Wang, R.Yang, L.Wang, Intelligent Multiagent Control for Integrated Building and Microgrid Systems. // IEEE PES Innovative Smart Grid Technologies (ISGT), Pp. 1-7, 2011.

[13] A. Celikyilmaz, I.B. Turksen, Enhanced fuzzy system models with improved fuzzy clustering algorithm. // IEEE Trans. Fuzzy Systems, Vol. 16, Pp. 779-794, 2008.

[14] Kazuo Tanaka, Hua O. Wang, "Fuzzy Control Systems Design and Analysis", Linear Matrix Inequality Approach / Copyright. - New York: John Wiley \& Sons, Inc., Pp.- 305, 2001.

[15] William Siler, James J. Buckley, "Fuzzy expert systems and fuzzy reasoning." Hoboken, NewJersey: John Wiley \& Sons, Inc., Pp. 405, 2005. 